\title{
Counting Closed Orbits for the Dyck Shift
}

\author{
Fahad Alsharari, Mohd Salmi Md. Noorani, and Habibulla Akhadkulov \\ School of Mathematical Sciences, Faculty of Science and Technology, Universiti Kebangsaan Malaysia, 43600 Bangi, Selangor, Malaysia
}

Correspondence should be addressed to Fahad Alsharari; fahd331@gmail.com

Received 27 February 2014; Accepted 29 September 2014; Published 12 November 2014

Academic Editor: Bernhard Ruf

Copyright (C) 2014 Fahad Alsharari et al. This is an open access article distributed under the Creative Commons Attribution License, which permits unrestricted use, distribution, and reproduction in any medium, provided the original work is properly cited.

The prime orbit theorem and Mertens' theorem are proved for a shift dynamical system of infinite type called the Dyck shift. Different and more direct methods are used in the proof without any complicated theoretical discussion.

\section{Introduction and Preliminaries}

In number theory, recall that, for functions $f, g: \mathbb{R} \rightarrow \mathbb{R}$, one says $f=O(g)$ means that $f(x)$ is bounded with respect to $g(x)$ for all sufficiently large $x$. That is, there exist constants $c$ and $x_{0}$ such that

$$
|f(x)|<c|g(x)|, \quad \forall x \geq x_{0},
$$

and $f(x)$ is asymptotic to $g(x)$, denoted by $f \sim g$, if

$$
\lim _{x \rightarrow \infty} \frac{f(x)}{g(x)}=1 \text {. }
$$

The prime-counting function that gives the number of primes less than or equal to $x$, for any real number $x$, is denoted by $\pi(x)$. The prime number theorem (PNT) is the statement

$$
\pi(x) \sim \frac{x}{\log (x)} .
$$

However, the asymptotic formula for primes $p$,

$$
\prod_{p \leq x}\left(1-\frac{1}{p}\right) \sim \frac{e^{-\gamma}}{\log x}
$$

where $\gamma=0.5772 \ldots$ is Euler's constant and $x \in \mathbb{R}$, is known as Mertens' theorem of analytic number theory. The logarithmic equivalent of Mertens' theorem is

$$
\sum_{p \leq x} \frac{1}{p}=\log \log x+B_{1}+O\left(\frac{1}{\log x}\right)
$$

and Mertens' constant $B_{1}=0.2614972128 \ldots$.
Let $X$ be a nonempty set and $T: X \rightarrow X$ a map. The pair $(X, T)$ is said to be a dynamical system. In techniques, a dynamical system is an abstract mathematical model describing the time dependence of point's position in its space. This is conventionally modeled by the map $T$ whose iterates denote the passage of time. Many dynamical questions involve counting the number of closed orbits or the periodic points under iteration of a map. A closed (periodic) orbit $\tau$ of length $|\tau|=n$ for a continuous map $T: X \rightarrow X$ is a set of the form $\left\{x, T x, T^{2} x, \ldots, T^{n-1} x\right\} \subset X$ where $T^{n} x=x$ for some $x \in X$ and $n \geq 1$.

Let $T: X \rightarrow X$ be a map, and define

$$
\begin{aligned}
& \mathscr{L}_{T}(n)=\left\{x \in X:\left|\left\{T^{k}(x)\right\}\right|_{k \in \mathbb{N}}=n\right\}, \\
& \mathscr{F}_{T}(n)=\left\{x \in X: T^{n}(x)=x\right\}, \\
& \mathcal{O}_{T}(n)=\{\tau: \tau \text { is a closed orbit of } T \text { of lengh }|\tau|=n\},
\end{aligned}
$$

which are the set of points of least period $n$ under $T$, the set of points of period $n$ under $T$, and the set of closed orbits of length $n$ under $T$, respectively. It is well known that the Möbius function, $\mu(n)$ of $n=1,2,3, \ldots$, is

$$
\mu(n)= \begin{cases}1 & \text { if } n=1 \\ (-1)^{k} & \text { if } n \text { is a product of } k \text { distinct primes; } \\ 0 & \text { otherwise. }\end{cases}
$$

Indeed, $|\mu(n)| \leq 1$ for any natural number $n$. Moreover, the sum over all positive divisors of $n$ of the Möbius function 
is zero except when $n=1$. The Möbius Inversion Formula [1] is defined as follows. If $f, g: \mathbb{N} \rightarrow \mathbb{C}$ are two arithmetic functions satisfying

$$
g(n)=\sum_{d \mid n} f(d), \quad \forall n,
$$

then

$$
f(n)=\sum_{d \mid n} \mu\left(\frac{n}{d}\right) g(d), \quad \forall n .
$$

Let

$$
\begin{gathered}
L_{T}(n)=\left|\mathscr{L}_{T}(n)\right|, \quad F_{T}(n)=\left|\mathscr{F}_{T}(n)\right|, \\
O_{T}(n)=\left|\mathcal{O}_{T}(n)\right| .
\end{gathered}
$$

It follows that

$$
\begin{gathered}
O_{T}(n)=\frac{L_{T}(n)}{n}, \\
F_{T}(n)=\sum_{d \mid n} L_{T}(d) .
\end{gathered}
$$

Consequently

$$
F_{T}(n)=\sum_{d \mid n} d O_{T}(d),
$$

and, hence, by Möbius Inversion Formula,

$$
O_{T}(n)=\frac{1}{n} \sum_{d \mid n} \mu\left(\frac{n}{d}\right) F_{T}(d) .
$$

Following the analogy between closed orbits and prime numbers, the asymptotic behavior of expressions like

$$
\pi_{T}(N)=|\{\tau:|\tau| \leq N\}|
$$

may be viewed as a dynamical analogue of the prime number theorem and a dynamical analogue of Mertens' theorem concerns asymptotic estimates for expressions like

$$
\mathscr{M}_{T}(N)=\sum_{n \leq N} \frac{O_{T}(n)}{e^{h n}},
$$

where $h$ denotes the topological entropy of the map $T$.

Parry in [2] initiated a line of research which uses ideas and techniques of analytic number theory to attack problems of this nature. When $X$ has a metric structure with respect to which $T$ is hyperbolic or shift of finite type (which will be given in detail in the subsequent section), results of Parry and Pollicott [3] and Noorani [4] have shown similar analogy between the number of closed orbits and the prime number theorem. It has been shown that

$$
\pi_{T}(N) \sim \frac{e^{h(N+1)}}{N\left(e^{h}-1\right)} .
$$

Sharp in [5] also obtained an analogy between the number of closed orbits and Mertens' theorem for hyperbolic maps as follows

$$
\mathscr{M}_{T}(N) \sim \log N+C_{1},
$$

for some constant $C_{1}$.

Several orbit-counting results on the asymptotic behavior of both (14) and (15) for other maps like quasihyperbolic toral automorphism (ergodic but not hyperbolic) can be found, for example, in [6-9].

In this paper, analogies between the number of closed orbits of a shift of infinite type called the Dyck shift and both (3) and (4) have been obtained. This paper is organized as follows. In the first section some introduction and preliminaries are given. In the second section the Dyck shift is introduced. The prime orbit theorem and Mertens' theorem are proved in Section 3.

\section{The Dyck Shift}

Let $\mathscr{A}$ be a finite alphabet. On $\mathscr{A}^{\mathbb{Z}}$ there acts the shift that sends the point $\left(x_{i}\right)_{i \in \mathbb{Z}} \in \mathscr{A}^{\mathbb{Z}}$ into the point $\left(x_{i+1}\right)_{i \in \mathbb{Z}} \in$ $\mathscr{A}^{\mathbb{Z}}$. The dynamical systems that are given by the closed shift invariant subsets of $\mathscr{A}^{\mathbb{Z}}$, with the restriction of the shift acting on them, are called subshifts. These are studied in symbolic dynamics. An element of $\mathscr{A}^{n}$ will be known as a word, or a block of length $n$. A word of length 0 is called an empty word and denoted by $\varepsilon$. The set of all finite words with letters taken from $\mathscr{A}$ is the set $\mathscr{A}^{*}=\bigcup_{n=0}^{\infty} \mathscr{A}^{n}$. A word is called admissible for the subshift $X \subset \mathscr{A}^{\mathbb{Z}}$ if it appears somewhere in a point of $X$. Let $X \subset \mathscr{A}^{\mathbb{Z}}$ and let $\mathscr{B}_{n}(X)$ denote the set of all admissible words of length $n$ in $X$. Then the language of $X$ is the collection $\mathscr{B}(X)=\bigcup_{n=0}^{\infty} \mathscr{B}_{n}(X)$. The topological entropy of a subshift $X \subset \mathscr{A}^{\mathbb{Z}}$ is given by

$$
h(X)=\lim _{n \rightarrow \infty} \frac{1}{n} \log \left|\mathscr{B}_{n}(X)\right| .
$$

Subshifts can be also defined using the notion of forbidden sets. Let $\mathscr{F}$ be a collection of words over $\mathscr{A}$, that is, $\mathscr{F} \subset \mathscr{A}^{*}$, which is called the forbidden set. For any such $\mathscr{F}$, define $X_{\mathscr{F}}$ to be the subset of sequences in $\mathscr{A}^{\mathbb{Z}}$ which do not contain any word in $\mathscr{F}$. Then, the subshift is a subset $X$ of a full shift $\mathscr{A}^{\mathbb{Z}}$ such that $X=\mathrm{X}_{\mathscr{F}}$ for some collection $\mathscr{F}$ of forbidden blocks over $\mathscr{A}$. If furthermore $\mathscr{F}$ is finite, then we call $X$ a subshift of finite type. The golden mean shift which is defined as the shift system over the alphabet $\mathscr{A}=\{0,1\}$ having a forbidden set $\mathscr{F}=\{11\}$ is a subshift of finite type.

The subshift comprises a lot of SFTs (shifts of finite type) in the shift space, which is said to have property A. A class of nonsofic systems, known as the Dyck systems, first suggested by Krieger [10] and named after an early contributor to the study of free groups and formal languages, codifies the rules of matching parentheses, which is one of these shift spaces. The Dyck shift $\mathfrak{D}_{N}$ which comes from language theory is defined to be the shift system over an alphabet $\mathscr{A}$ that consists of $N$ negative symbols and $N$ positive symbols. For an $x$ in the full shift $\mathscr{A}^{\mathbb{Z}}, x$ is in $\mathfrak{D}_{N}$ if and only if every finite 
block appearing in $x$ has a nonzero reduced form. Therefore, the constraint for $x$ cannot be bounded. A beautiful way to describe the Dyck shift is in terms of its syntactic monoid.

Let $\mathscr{A}=\left\{\ell_{1}, \ell_{2}, \ldots, \ell_{M}, r_{1}, r_{2}, \ldots, r_{M}\right\}$. The alphabet consists of $M$ pairs of matching left and right delimiters or symbols. Let $\mathscr{M}$ be a monoid (with zero) with generators $\ell_{i}, r_{i}$, $1 \leq i \leq M$, and 1 . The relations on the monoid are

$$
\begin{gathered}
\ell_{i} \circ r_{j}=1 \quad \text { if } i=j, 1 \leq i, j \leq M, \\
\ell_{i} \circ r_{j}=0 \quad \text { if } i \neq j, \quad 1 \leq i, j \leq M, \\
\alpha \circ 1=1 \circ \alpha=\alpha, \quad \alpha \in \mathscr{A} \cup\{1\}, \\
\alpha \circ 0=0 \circ \alpha=0, \quad \alpha \in \mathscr{A} \cup\{1\}, \\
0 \circ 0=0 .
\end{gathered}
$$

We use a mapping $\operatorname{red}(): \mathscr{A}^{*} \rightarrow \mathscr{M}$ such that, for

$$
\begin{gathered}
\omega=\omega_{1} \omega_{2} \cdots \omega_{n} \in \mathscr{A}^{*} \quad(n \geq 1), \\
\operatorname{red}(\omega)=\omega_{1} \circ \omega_{2} \circ \cdots \circ \omega_{n}, \quad \operatorname{red}(\varepsilon)=1 .
\end{gathered}
$$

Definition 1. The Dyck shift $\mathfrak{D}_{M}[10]$ is defined by

$$
\mathfrak{D}_{M}=\left\{x \in \mathscr{A}^{\mathbb{Z}}: \text { if } i \leq j \text {, then } \operatorname{red}\left(x_{[i, j)}\right) \neq 0\right\},
$$

where $x_{[i, j)}=x_{i} x_{i+1} \cdots x_{j-1}$.

When $M=1, \mathfrak{D}_{1}$ is the full shift on two symbols; we will tacitly assume that $M \geq 2$. The topological entropy of the Dyck shift $\mathfrak{D}_{M}$ is already computed as $\log (M+1)$ in [10].

Theorem 2 (see [11]). The number of points in the Dyck shift $\mathfrak{D}_{M}$ having period $n$ is given by

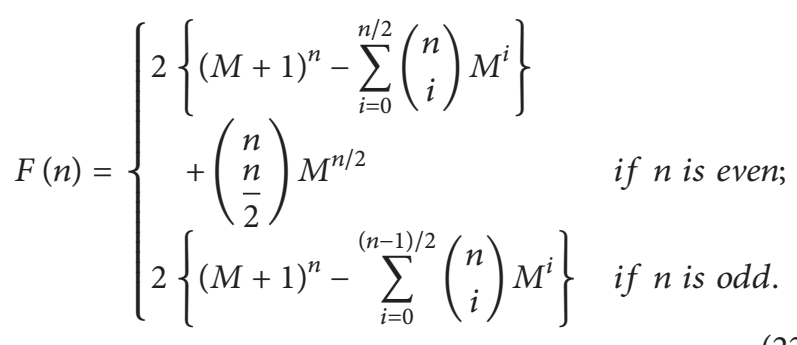

\section{Counting Closed Orbits}

In this section, we prove two theorems that involve the counting of orbits for the Dyck shift, where the first one is the prime orbit theorem and the second one is Mertens' orbit theorem. However, in order to prove these theorems, we firstly prove the following lemma which plays an important role in proving our main results.

Lemma 3. There exist constants $0<c_{1}<1$ and $c_{2}>1$ such that the following inequality holds for all $n \geq 1$ :

$$
c_{1}(M+1)^{n} \leq F(n) \leq c_{2}(M+1)^{n} .
$$

Proof. Assume that $n$ is even. The case where $n$ is odd can be proved analogously.

Note first that

$$
\sum_{i=0}^{n / 2}\left(\begin{array}{c}
n \\
i
\end{array}\right) M^{i}<(M+1)^{n}, \quad\left(\begin{array}{c}
n \\
\frac{n}{2}
\end{array}\right) M^{n / 2}<(M+1)^{n} .
$$

Therefore, we obtain

$$
\begin{aligned}
F(n) & =2(M+1)^{n}\left\{1-\frac{\sum_{i=0}^{n / 2}\left(\begin{array}{c}
n \\
i
\end{array}\right) M^{i}}{(M+1)^{n}}+\frac{\left(\begin{array}{c}
n \\
n / 2
\end{array}\right) M^{n / 2}}{(M+1)^{n}}\right\} \\
& <3(M+1)^{n}, \quad \forall n \geq 1 .
\end{aligned}
$$

Also

$$
\begin{aligned}
F(n) & \geq(M+1)^{n}-\sum_{i=0}^{n / 2}\left(\begin{array}{c}
n \\
i
\end{array}\right) M^{i}+\left(\begin{array}{c}
n \\
\frac{n}{2}
\end{array}\right) M^{n / 2} \\
& =(M+1)^{n}\left[1-\frac{\sum_{i=0}^{n / 2}\left(\begin{array}{c}
n \\
i
\end{array}\right) M^{i}}{(M+1)^{n}}\right] .
\end{aligned}
$$

Estimating the term $\left[1-\sum_{i=0}^{n / 2}\left(\begin{array}{c}n \\ i\end{array}\right) M^{i} /(M+1)^{n}\right]$ using the binomial theorem we obtain

$$
\begin{aligned}
& \frac{\sum_{i=0}^{n / 2}\left(\begin{array}{c}
n \\
i
\end{array}\right) M^{i}}{(M+1)^{n}} \\
& =\frac{\sum_{i=0}^{n / 2}\left(\begin{array}{c}
n \\
i
\end{array}\right) M^{i}}{\sum_{i=0}^{n / 2-1}\left(\begin{array}{c}
n \\
i
\end{array}\right) M^{i}+\left(\begin{array}{c}
n \\
n / 2
\end{array}\right) M^{n / 2}+\sum_{i=n / 2+1}^{n}\left(\begin{array}{c}
n \\
i
\end{array}\right) M^{i}} \\
& \leq \frac{\sum_{i=0}^{n / 2}\left(\begin{array}{c}
n \\
i
\end{array}\right) M^{i}}{\sum_{i=0}^{n / 2-1}\left(\begin{array}{c}
n \\
i
\end{array}\right) M^{i}+\sum_{i=n / 2+1}^{n}\left(\begin{array}{c}
n \\
i
\end{array}\right) M^{i}} .
\end{aligned}
$$

We set

$$
\begin{aligned}
& \mathrm{A}_{n}:=\sum_{i=0}^{n / 2-1}\left(\begin{array}{l}
n \\
i
\end{array}\right) M^{i}=\sum_{i=0}^{n / 2-1}\left(\begin{array}{c}
n \\
\frac{n}{2}-i-1
\end{array}\right) M^{n / 2-i-1}, \\
& \mathrm{~B}_{n}:=\sum_{i=n+1}^{n}\left(\begin{array}{l}
n \\
i
\end{array}\right) M^{i}=\sum_{i=0}^{n / 2-1}\left(\begin{array}{c}
n \\
\frac{n}{2}+i+1
\end{array}\right) M^{n / 2+i+1} ;
\end{aligned}
$$

using the definition of the binomial coefficient it is easy to prove that

$$
\left(\begin{array}{c}
n \\
\frac{n}{2}-i-1
\end{array}\right)=\left(\begin{array}{c}
n \\
\frac{n}{2}+i+1
\end{array}\right) \quad \text { for any } 0 \leq i \leq \frac{n}{2}-1 \text {, }
$$

and also

$$
M^{n / 2+i+1} \geq M^{2}\left(M^{n / 2-i-1}\right) \quad \text { for any } 0 \leq i \leq \frac{n}{2}-1
$$

utilizing (29) and (30) we obtain

$$
\mathrm{B}_{n} \geq M^{2}\left(\mathrm{~A}_{n}\right) .
$$


Applying inequality (31) to (27), we get

$$
\frac{\sum_{i=0}^{n / 2}\left(\begin{array}{c}
n \\
i
\end{array}\right) M^{i}}{(M+1)^{n}} \leq \frac{\mathrm{A}_{n}}{\mathrm{~A}_{n}+\mathrm{B}_{n}} \leq \frac{\mathrm{A}_{n}}{\mathrm{~A}_{n}+M^{2}\left(\mathrm{~A}_{n}\right)}=\frac{1}{1+M^{2}}
$$

hence, inequality (26) implies that

$$
F(n) \geq(M+1)^{n}\left(1-\frac{1}{1+M^{2}}\right)=\frac{M^{2}}{1+M^{2}}(M+1)^{n} .
$$

Thus, if we take $c_{1}=M^{2} /\left(1+M^{2}\right)$ and $c_{2}=3$, then we are done.

Theorem 4 (prime orbit theorem). Let $T: \mathfrak{D}_{M} \rightarrow \mathfrak{D}_{M}$ be the Dyck shift on a set of $M$ pairs and $\tau$ a closed orbit for $T$. Let $\pi(N)$ be the number of closed orbits of $T$ not exceeding $N$ and $F(n)$ the number of points having period $n$ under $T$ as given by Theorem 2. Then

$$
\pi(N) \sim \frac{F(N)}{N^{3 / 4}} .
$$

Proof. Using Lemma 3, we get

$$
\begin{aligned}
\sum_{n \leq N} F(n) & \leq c_{2} \sum_{n \leq N}(M+1)^{n} \\
& =c_{2}(M+1)^{N}\left(\sum_{n \leq N}(M+1)^{n-N}\right) \\
& \leq c_{2} \frac{1}{1-1 /(M+1)}(M+1)^{N} \\
& =\frac{c_{2}(M+1)}{M}(M+1)^{N} \\
& \leq \frac{c_{2}(M+1)}{c_{1} \cdot M} F(N) .
\end{aligned}
$$

Similarly,

$$
\begin{aligned}
\sum_{n \leq N} F(n) & \geq c_{1} \sum_{n \leq N}(M+1)^{n} \\
& =c_{1}(M+1)^{N}\left(\sum_{n \leq N}(M+1)^{n-N}\right) \\
& \geq \frac{c_{1}}{c_{2}}\left(\sum_{n \leq N}(M+1)^{n-N}\right) F(N) ;
\end{aligned}
$$

by combining (35) and (36), we obtain

$$
0<\frac{c_{1}(M+1)}{c_{2} \cdot M} \leq \lim _{N \rightarrow \infty} \frac{\sum_{n \leq N} F(n)}{F(N)} \leq \frac{c_{2}(M+1)}{c_{1} \cdot M}<\infty .
$$

Thus, by Möbius Inversion Formula we have

$$
\begin{aligned}
\pi(N) & =\sum_{n \leq N} \frac{1}{n} \sum_{d \mid n} \mu\left(\frac{n}{d}\right) F(d) \\
& =\sum_{n \leq N} \frac{1}{n} \sum_{d \mid n, d<n} \mu\left(\frac{n}{d}\right) F(d)+\sum_{n \leq N} \frac{1}{n} F(n) .
\end{aligned}
$$

Subtracting the dominant terms, we obtain

$$
\begin{aligned}
\left|\pi(N)-\sum_{n \leq N} \frac{1}{n} F(n)\right| & \leq\left|\sum_{n \leq N} \frac{1}{n} \sum_{d \mid n, d<n} \mu\left(\frac{n}{d}\right) F(d)\right| \\
& \leq \sum_{n \leq N} \frac{1}{n} \sum_{d \mid n, d<n} F(d) \\
& \leq \sum_{n \leq N} \sum_{d \mid n, d<n} F(d)
\end{aligned}
$$

since $d \mid n, d<n$, then $d \leq\lfloor n / 2\rfloor$, so

$$
\text { equation (39) } \begin{aligned}
\leq & \sum_{n \leq N} \sum_{d \leq\lfloor n / 2\rfloor} F(d) \\
& \leq \sum_{n \leq N} F\left(\frac{n}{2}\right) \\
& =O\left(F\left(\frac{N}{2}\right)\right)
\end{aligned}
$$

To estimate the dominant terms, let $K(N)=\left\lfloor N^{1 / 4}\right\rfloor$. Then

$$
\begin{aligned}
\left|\sum_{n \leq N} \frac{1}{n} F(n)-\sum_{N-K(N) \leq n \leq N} \frac{1}{n} F(n)\right| & \leq \sum_{n \leq N-K(N)} F(n) \\
& =O(F(N-K(N))) .
\end{aligned}
$$

Finally

$$
\begin{aligned}
& \sum_{N-K(N) \leq n \leq N} \frac{1}{n} F(n) \\
& =\frac{F(N)}{N}\left[\sum_{r=0}^{K(N)}\left(\frac{F(N-r)}{F(N)} \cdot \frac{N}{N-r}\right)\right] \\
& =\frac{F(N)}{N}\left[\sum_{r=0}^{K(N)}\left(\frac{F(N-r)}{F(N)} \cdot\left(\sum_{n=0}^{\infty}\left(\frac{r}{N}\right)^{n}\right)\right)\right] \\
& =\frac{F(N)}{N}\left[\sum _ { r = 0 } ^ { K ( N ) } \left(\frac{F(N-r)}{F(N)}+\frac{F(N-r)}{F(N)}\left(\frac{r}{N}\right)\right.\right. \\
& \left.\left.+\frac{F(N-r)}{F(N)}\left(\frac{r}{N}\right)^{2}+\cdots\right)\right]
\end{aligned}
$$

in fact

$$
\frac{F(N-r)}{F(N)} \leq 1, \quad \forall 0 \leq r \leq K(N)
$$


then

$$
\begin{aligned}
\text { equation }(42) \leq & \frac{F(N)}{N}\left[\sum _ { r = 0 } ^ { K ( N ) } \left(\frac{F(N-r)}{F(N)}+\left(\frac{r}{N}\right)+\left(\frac{r}{N}\right)^{2}\right.\right. \\
& \left.\left.+\left(\frac{r}{N}\right)^{3}+\cdots\right)\right] \\
= & \frac{F(N)}{N}\left[\sum_{r=0}^{K(N)} \frac{F(N-r)}{F(N)}+\sum_{r=0}^{K(N)} \sum_{j=1}^{\infty}\left(\frac{r}{N}\right)^{j}\right] \\
\leq & \frac{F(N)}{N}\left[\sum_{r=0}^{K(N)} 1+\sum_{r=0}^{K(N)} \sum_{j=1}^{\infty}\left(\frac{r}{N}\right)^{j}\right] ;
\end{aligned}
$$

$\sum_{j=1}^{\infty}(r / N)^{j}$ is a geometric series with $(r / N)$ as a first term; then we have

$$
\begin{aligned}
& =\frac{F(N)}{N}\left[\sum_{r=0}^{K(N)} 1+O\left(\sum_{r=0}^{K(N)} \frac{r}{N}\right)\right] \\
& =\frac{F(N)}{N}\left[(K(N)+1)+O\left(\sum_{r=0}^{K(N)} \frac{r}{N}\right)\right] \\
& =\left[\frac{(F(N))(K(N)+1)}{N}+O\left(\frac{(F(N)) \sum_{r=0}^{K(N)} r}{N^{2}}\right)\right] \\
& =\frac{(F(N))(K(N)+1)}{N}+O\left(\frac{F(N)}{N^{3 / 2}}\right),
\end{aligned}
$$

since $K(N)=\left\lfloor N^{1 / 4}\right\rfloor$.

Therefore, it can be seen that from (40), (41), and (45)

$$
\begin{gathered}
\pi(N)-\sum_{n \leq N} \frac{F(n)}{n} \leq k_{1}\left(F\left(\frac{N}{2}\right)\right), \\
\sum_{n \leq N} \frac{F(n)}{n}-\sum_{N-K(N) \leq n \leq N} \frac{F(n)}{n} \leq k_{2}(F(N-K(N))), \\
\sum_{N-K(N) \leq n \leq N} \frac{F(n)}{n}-\left(\frac{(F(N))(K(N)+1)}{N}\right) \leq k_{3}\left(\frac{F(N)}{N^{3 / 2}}\right) .
\end{gathered}
$$

Thus

$$
\begin{aligned}
& \pi(N)-\left(\frac{(F(N))(K(N)+1)}{N}\right) \\
& \leq k_{1}\left(F\left(\frac{N}{2}\right)\right)+k_{2}(F(N-K(N)))+k_{3}\left(\frac{F(N)}{N^{3 / 2}}\right) \\
& \Longrightarrow \pi(N) \cdot\left(\frac{N}{(F(N))(K(N)+1)}\right)-1
\end{aligned}
$$

$$
\begin{aligned}
\leq & k_{1}\left(\frac{(N)(F(N / 2))}{(F(N))(K(N)+1)}\right) \\
& +k_{2}\left(\frac{(N)(F(N-K(N)))}{(F(N))(K(N)+1)}\right) \\
& +k_{3}\left(\frac{(N)(F(N))}{\left(N^{3 / 2}\right)(F(N))(K(N)+1)}\right) ;
\end{aligned}
$$

since $K(N)=\left\lfloor N^{1 / 4}\right\rfloor \Rightarrow K(N) \leq N^{1 / 4}$, then

$$
\begin{aligned}
\pi(N) & \cdot\left(\frac{N}{(F(N))\left(N^{1 / 4}+1\right)}\right)-1 \\
\leq & k_{1}\left(\frac{(N)(F(N / 2))}{(F(N))\left(N^{1 / 4}+1\right)}\right) \\
& +k_{2}\left(\frac{(N)\left(F\left(N-N^{1 / 4}\right)\right)}{(F(N))\left(N^{1 / 4}+1\right)}\right) \\
& +k_{3}\left(\frac{(N)(F(N))}{\left(N^{3 / 2}\right)(F(N))\left(N^{1 / 4}+1\right)}\right) .
\end{aligned}
$$

Using Lemma 3, we obtain

$$
\begin{aligned}
& \frac{N(F(N / 2))}{F(N)\left(N^{1 / 4}+1\right)} \leq \frac{N^{3 / 4} \cdot c_{2}(M+1)^{N / 2}}{c_{1} \cdot(M+1)^{N}} \\
&=\frac{N^{3 / 4} \cdot c_{2}}{c_{1} \cdot(M+1)^{N / 2}} \longrightarrow 0 \quad \text { as } N \longrightarrow \infty, \\
& \frac{N\left(F\left(N-N^{1 / 4}\right)\right)}{F(N)\left(N^{1 / 4}+1\right)} \leq \frac{N^{3 / 4} \cdot c_{2}}{c_{1} \cdot(M+1)^{N^{1 / 4}}} \longrightarrow 0 \\
& \text { as } N \longrightarrow \infty .
\end{aligned}
$$

Thus, letting $N \rightarrow \infty$ implies that

$$
\begin{aligned}
& k_{1}\left(\frac{(N)(F(N / 2))}{(F(N))\left(N^{1 / 4}+1\right)}\right)+k_{2}\left(\frac{(N)\left(F\left(N-N^{1 / 4}\right)\right)}{(F(N))\left(N^{1 / 4}+1\right)}\right) \\
& \quad+k_{3}\left(\frac{(N)(F(N))}{\left(N^{3 / 2}\right)(F(N))\left(N^{1 / 4}+1\right)}\right) \rightarrow 0 .
\end{aligned}
$$

So

$$
\pi(N) \cdot\left(\frac{N}{(F(N))\left(N^{1 / 4}+1\right)}\right)-1 \longrightarrow 0 .
$$

Therefore

$$
\pi(N) \sim \frac{(F(N))\left(N^{1 / 4}+1\right)}{N},
$$

which is equivalent to

$$
\pi(N) \sim \frac{F(N)}{N^{3 / 4}},
$$

as required. 
Theorem 5 (Mertens' orbit theorem). Let $T: \mathfrak{D}_{M} \rightarrow \mathfrak{D}_{M}$ be the Dyck shift on a set of $M$ pairs and $F(n)$ the number of points having period $n$ under $T$ that is given in Theorem 2. Then

$$
\begin{aligned}
& \mathscr{M}_{T}(N) \\
& \quad=O\left(F(N)\left[\frac{1-e^{-h N}}{e^{h}-1}-\log \left(1-\frac{1}{e^{h}}\right)+\left(N^{-1}\right)\right]\right),
\end{aligned}
$$

where $h=\log (M+1)$.

Proof. The dynamical Mertens' theorem asserts that

$$
\begin{aligned}
\mathscr{M}_{T}(N) & =\sum_{n \leq N} \frac{O(n)}{e^{h n}} \\
& =\sum_{n \leq N} \frac{1}{n e^{h n}} \sum_{d \mid n} \mu\left(\frac{n}{d}\right) F(d) \\
& =\sum_{n \leq N} \frac{1}{n e^{h n}} \sum_{d \mid n, d<n} \mu\left(\frac{n}{d}\right) F(d)+\sum_{n \leq N} \frac{F(n)}{n e^{h n}} .
\end{aligned}
$$

Firstly we want to estimate the term

$$
\sum_{n \leq N} \frac{1}{n e^{h n}} \sum_{d \mid n, d<n} \mu\left(\frac{n}{d}\right) F(d) .
$$

Note that

$$
|\mu(n)| \leq 1, \quad \sum_{d \mid n, d<n} F(d) \leq n F\left(\frac{n}{2}\right) \quad \forall n \in \mathbb{N} .
$$

Hence

$$
\begin{aligned}
& \left|\sum_{n=1}^{N} \frac{1}{n e^{h n}} \sum_{d \mid n, d<n} \mu\left(\frac{n}{d}\right) F(d)\right| \\
& \leq\left|\sum_{n=1}^{N} \frac{1}{n e^{h n}} \cdot n F\left(\frac{n}{2}\right)\right|=\sum_{n=1}^{N} \frac{F(n / 2)}{e^{h n}} \\
& \quad \leq \sum_{n=1}^{N} \frac{F(N / 2)}{e^{h n}} \\
& =F\left(\frac{N}{2}\right) \cdot \sum_{n=1}^{N} \frac{1}{e^{h n}}(\text { is a partial sum of a geometric series }) \\
& \quad=F\left(\frac{N}{2}\right) \cdot\left(\frac{e^{-h}-\left(e^{-h}\right)^{N+1}}{1-e^{-h}}\right) \\
& \leq F(N) \cdot\left(\frac{e^{-h}-\left(e^{-h}\right)^{N+1}}{1-e^{-h}}\right)
\end{aligned}
$$

therefore

$$
\sum_{n=1}^{N} \frac{1}{n e^{h n}} \sum_{d \mid n, d<n} \mu\left(\frac{n}{d}\right) F(d)=O\left(\frac{F(N) \cdot\left(1-e^{-h N}\right)}{e^{h}-1}\right) .
$$

Now for

$$
\sum_{n \leq N} \frac{F(n)}{n e^{h n}}
$$

in order to estimate this partial sum, the following lemma is extremely needed.

Lemma 6. If $\omega$ is a complex number of modulus one and is not a root of unity, then

$$
\sum_{n \leq N} \frac{\omega^{n}}{n}=-\log (1-\omega)+O\left(N^{-1}\right) .
$$

Since $h>0$, then $1 / e^{h} \neq 1$; therefore we can apply the above lemma. Hence

$$
\begin{aligned}
\sum_{n \leq N} \frac{F(n)}{n e^{h n}} & \leq \sum_{n \leq N} \frac{F(N)}{n e^{h n}} \\
& =F(N)\left[-\log \left(1-\frac{1}{e^{h}}\right)+O\left(N^{-1}\right)\right] .
\end{aligned}
$$

It follows that

$$
\begin{aligned}
& \mathscr{M}_{T}(N) \\
& \quad=O\left(F(N)\left[\frac{1-e^{-h N}}{e^{h}-1}-\log \left(1-\frac{1}{e^{h}}\right)+O\left(N^{-1}\right)\right]\right) .
\end{aligned}
$$

In fact, from the properties of asymptotic estimates, we have

$$
O(O(f(x)))=O(f(x)) .
$$

Therefore, we conclude that

$$
\begin{aligned}
& \mathscr{M}_{T}(N) \\
& \quad=O\left(F(N)\left[\frac{1-e^{-h N}}{e^{h}-1}-\log \left(1-\frac{1}{e^{h}}\right)+\left(N^{-1}\right)\right]\right) .
\end{aligned}
$$

\section{Conflict of Interests}

The authors declare that there is no conflict of interests regarding the publication of this paper.

\section{Acknowledgments}

The authors are grateful to Universiti Kebangsaan Malaysia for providing the financial support via the Grant no. FRGS/1/2014/ST06/UKM/01/1 and Model Insan Berpusat (NIC1). The authors thank the referee for his/her careful reading of the paper and useful suggestions.

\section{References}

[1] G. H. Hardy and E. M. Wright, An Introduction to the Theory of Numbers, Oxford University Press, Oxford, UK, 1938. 
[2] W. Parry, "An analogue of the prime number theorem for closed orbits of shifts of finite type and their suspensions," Israel Journal of Mathematics, vol. 45, no. 1, pp. 41-52, 1983.

[3] W. Parry and M. Pollicott, "An analogue of the prime number theorem for closed orbits of Axiom A Flows," Annals of Mathematics, vol. 118, no. 3, pp. 573-591, 1983.

[4] M. S. M. Noorani, "Counting closed orbits of hyperbolic diffeomorphisms," Results in Mathematics, vol. 50, no. 3-4, pp. 241257, 2007.

[5] R. Sharp, "An analogue of Mertens'theorem for closed orbits of Axiom A flows," Boletim da Sociedade Brasileira de Matemática. Nova Série, vol. 21, no. 2, pp. 205-229, 1991.

[6] S. Waddington, "The prime orbit theorem for quasihyperbolic toral automorphisms," Monatshefte für Mathematik, vol. 112, no. 3, pp. 235-248, 1991.

[7] M. S. Noorani, "Mertens theorem and closed orbits of ergodic toral automorphisms," The Bulletin of the Malaysian Mathematical Society Series, vol. 22, no. 2, pp. 127-133, 1999.

[8] S. Jaidee, S. Stevens, and T. Ward, "Mertens' theorem for toral automorphisms," Proceedings of the American Mathematical Society, vol. 139, no. 5, pp. 1819-1824, 2011.

[9] G. Everest, R. Miles, S. Stevens, and T. Ward, "Orbit-counting in non-hyperbolic dynamical systems," Journal für die Reine und Angewandte Mathematik, vol. 608, pp. 155-182, 2007.

[10] W. Krieger, "On the uniqueness of the equilibrium state," Mathematical Systems Theory, vol. 8, no. 2, pp. 97-104, 1974.

[11] T. Hamachi and K. Inoue, "Embedding of shifts of finite type into the Dyck shift," Monatshefte für Mathematik, vol. 145, no. 2, pp. 107-129, 2005. 


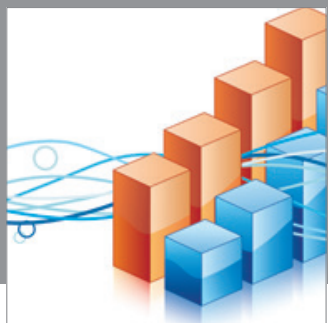

Advances in

Operations Research

mansans

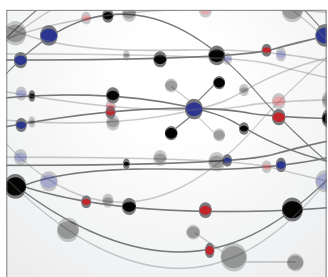

The Scientific World Journal
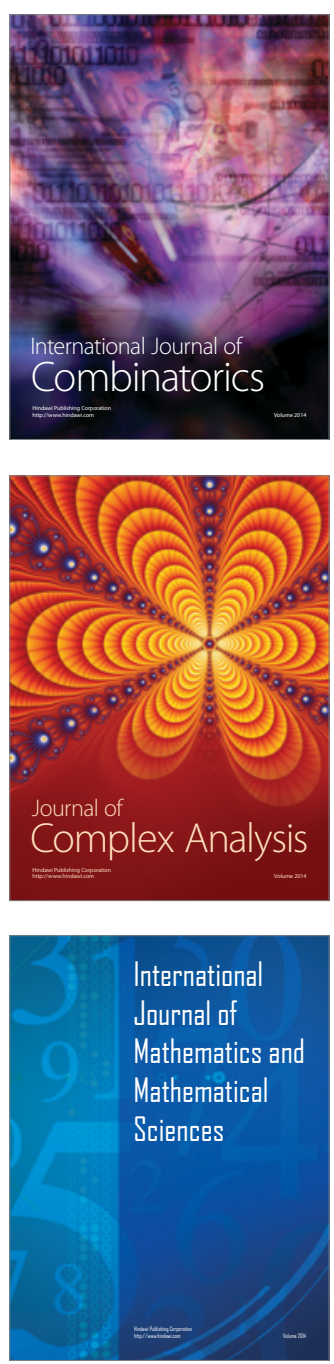
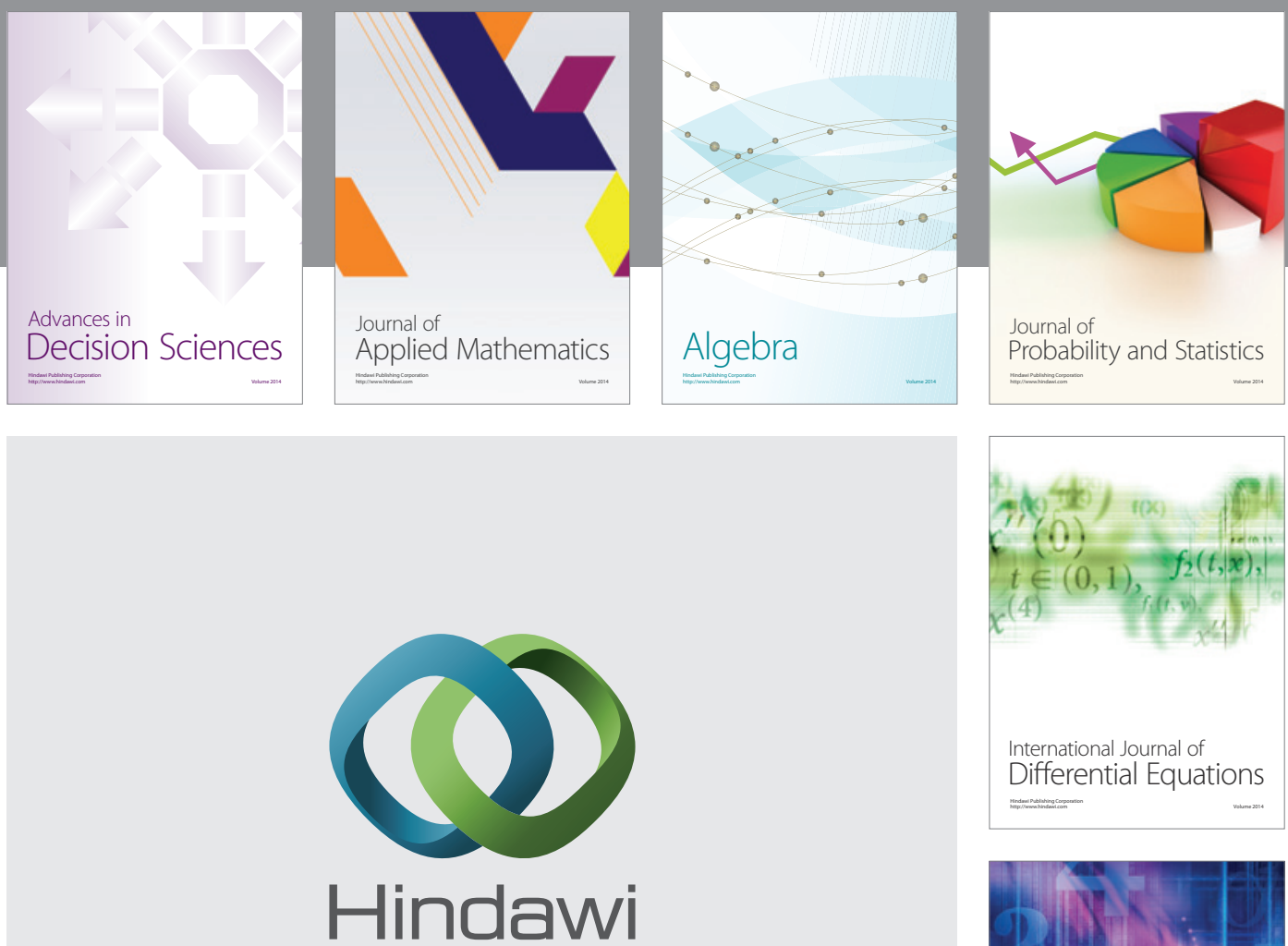

Submit your manuscripts at http://www.hindawi.com
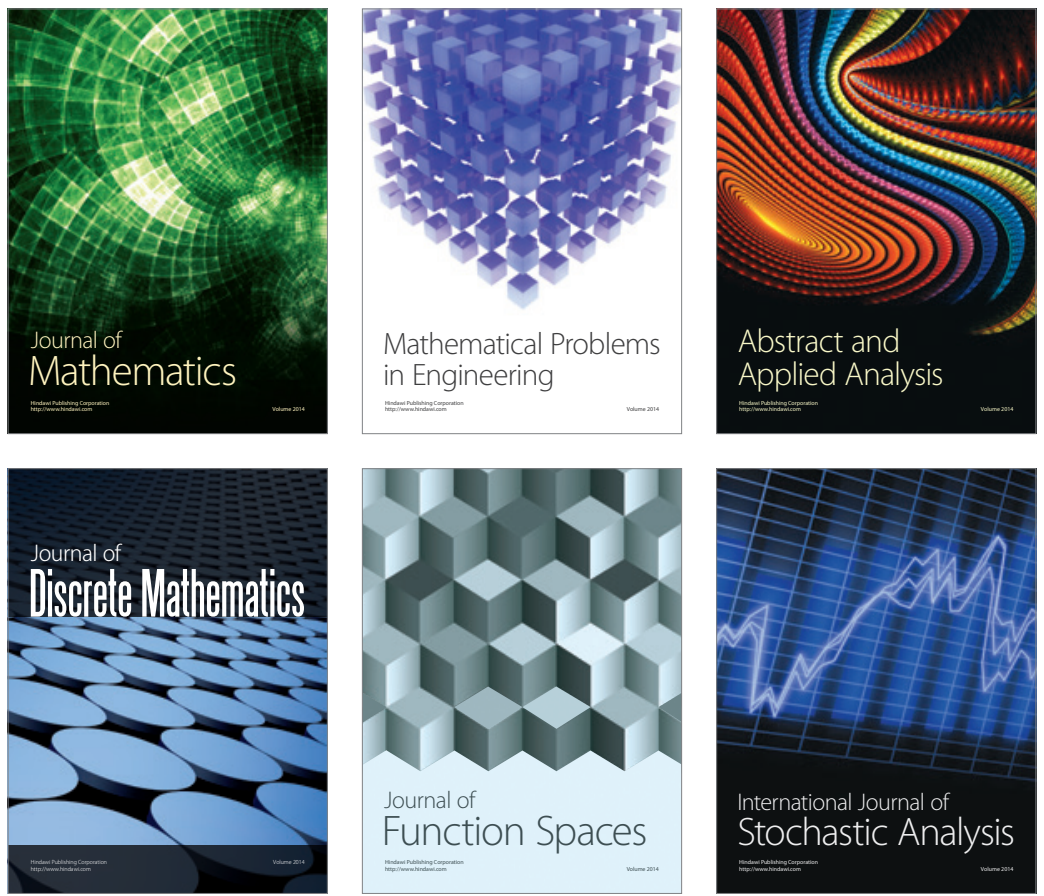

Journal of

Function Spaces

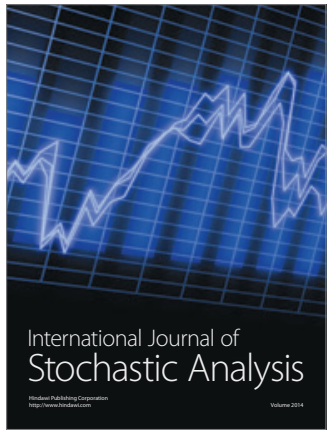

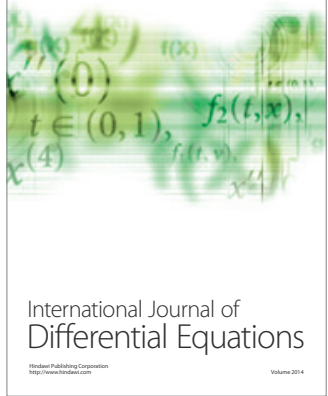
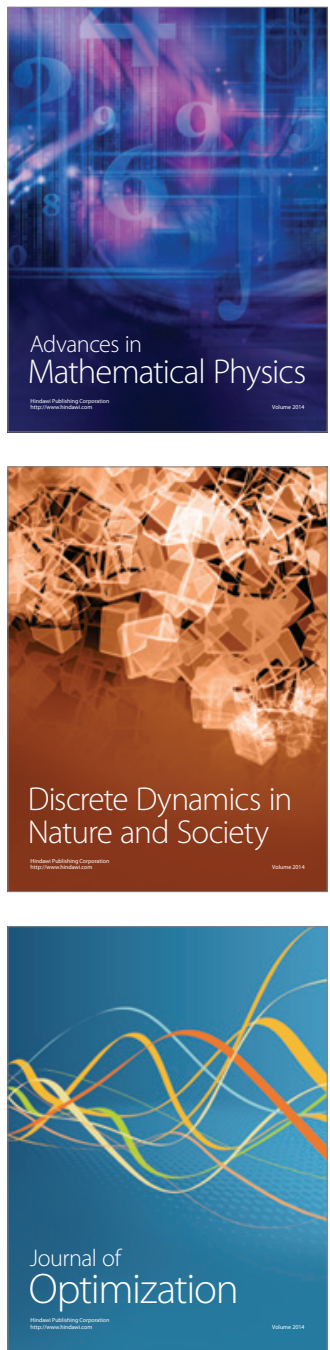